\title{
Different perspectives and viewpoints on the postoperative management of EGFR-mutant lung cancer
}

\author{
Masayuki Nakao, Junji Ichinose, Yosuke Matsuura, Sakae Okumura, Mingyon Mun \\ Department of Thoracic Surgical Oncology, Cancer Institute Hospital, The Japanese Foundation for Cancer Research, Tokyo, Japan \\ Correspondence to: Masayuki Nakao, MD. Cancer Institute Hospital, 3-10-6 Ariake, Koto-ku, Tokyo 135-8550, Japan. \\ Email: masayuki.nakao@jfcr.or.jp. \\ Comment on: Liang W, Cai K, Chen C, et al. Society for Translational Medicine consensus on postoperative management of EGFR-mutant lung \\ cancer (2019 edition). Transl Lung Cancer Res 2019;8:1163-73.
}

Submitted Mar 21, 2020. Accepted for publication Apr 03, 2020.

doi: $10.21037 / \mathrm{atm} .2020 .04 .34$

View this article at: http://dx.doi.org/10.21037/atm.2020.04.34

Despite the era of immune-oncology for lung cancer, epidermal growth factor receptor (EGFR) mutation is the critical target of treatment. EGFR mutations have been identified in $47.9 \%$ of the adenocarcinomas and $4.6 \%$ of the squamous cell carcinomas diagnosed in Asian patients as well as in $19.2 \%$ of the adenocarcinomas and $3.3 \%$ of the squamous cell carcinomas diagnosed in Western patients (1). EGFR-mutant non-small cell lung cancer (NSCLC) requires specific treatment strategies that include EGFRtyrosine kinase inhibitors (TKIs). TKIs are now used routinely in the management of EGFR-mutant NSCLCs, including patients with advanced disease and also those who have been treated with surgical resection.

Liang et al. have recently provided a consensus statement on the postoperative management of lung cancer associated with EGFR mutations on behalf of National Clinical Research Center for Respiratory Disease, Thoracic Surgery Branch of China International Exchange and Promotion Association for Medical and Healthcare, Chinese Alliance Against Lung Cancer and AME Thoracic Surgery Collaborative Group (2). The statement summarizes eight specific recommendations using a four-point scale to emphasize the strength of currently-available evidence. Experts in the field from outside China were invited to comment on several controversial issues. In consideration of these comments, we are reminded that there are different perspectives and viewpoints on postoperative management of lung cancer with EGFR mutations.

Consensus statement \#1 is as follows: "detection of EGFR mutations is routinely recommended in surgically resected specimens of non-squamous NSCLC (level of evidence: $2 A$; strength of recommendation: strong)." This recommendation is focuses on the possibility of effective adjuvant treatment including EGFR-TKIs for patients with surgically resected NSCLCs with EGFR mutations. However, discussed below, there is controversy regarding the efficacy of adjuvant treatments using EGFR-TKIs in these circumstances and under these conditions. With regard to treatment of recurrence, there is no question that EGFR-TKIs are the most promising drug for recurrent disease after surgical resection. Although one would certainly make and attempt to re-biopsy any recurrent disease, this is not always possible given the physical condition of the patient and the location of the lesion. Similarly, stored surgical specimens may deteriorate to the point where accurate detection of gene mutations is impossible. As such, it makes sense to proceed with strategies to detect EGFR mutations in resected specimens immediately, especially for patients at high risk for relapse. Another consideration focuses on the expenses involved with respect to the detection and identification of EGFR mutations. For example, real time PCR assays performed on formalin-fixed paraffin-embedded tissues currently costs about $\$ 200$ (U.S. dollars) in Japan. From an economic standpoint, it is clearly not advisable to evaluate EGFR mutations in patients who are at minimal risk for relapse, such as those with adenocarcinoma in situ, minimally invasive adenocarcinoma, or lepidic predominant adenocarcinoma.

Consensus statement \#3 continues: "for patients with EGFR mutations, EGFR-TKI can be used as one of the postoperative adjuvant treatment options for patients with stage II-IIIA EGFR-mutant NSCLC, especially for those patients 
at a high risk of recurrence and with poor expected tolerance to chemotherapy (level of evidence: 1 ; strength of recommendation: strong)." The authors report that EGFR-TKIs have become an important option for postoperative adjuvant treatment for patients with EGFR-mutant NSCLC. This recommendation was based on results from several randomized clinical trials including the RADIANT, ADJUVANT, and EVAN studies (3-5). However, as Expert Opinion 5 notes, while these trials clearly indicate a benefit with respect to disease free survival (DFS), they did not indicate significant benefits with respect to overall survival (OS). Among these reports, the ADJUVANT study is most noteworthy because it is the only head to head comparison of gefitinib $v s$. vinorelbine plus cisplatin as adjuvant treatment for stage II-IIIA EGFR-mutant NSCLC that included over 200 patients. Significant increases in DFS were definitively demonstrated among those in the gefitinib group (hazard ratio: 0.60); ultimately, this result may translate into improved OS as well. However, the study design does include post-recurrence treatment with EGFRTKIs for the patients treated with vinorelbine plus cisplatin. If gefitinib simply delays disease recurrence, its role in promoting OS will remain indeterminate. A prospective randomized trial, WJOG 6410L, with almost the same design with the ADJUVANT study, is ongoing in Japan. Patient accrual was completed in 2015 and we all anticipate the results of this study with great interest. However, at this point, it may not be useful to include EGFR-TKIs as standard postoperative adjuvant treatment for patients with EGFR-mutant NSCLC until there are proven benefits with respect to OS, although these agents may be an option for the patients who are unable to tolerate standard cytotoxic chemotherapy. The use of these agents may depend on the circumstances of individual patients and may be decided on a case-by-case basis. At this time, adjuvant use of EGFRTKIs is not covered by medical insurance in Japan.

Consensus statement 6 states: "annual brain MRI and bone scans in addition to regular chest CT are recommended for EGFRmutant NSCLC, and the scan frequencies can be increased in patients at a bigh risk for recurrence (level of evidence: $2 A$; strength of recommendation: strong)." There are only retrospective analyzes and older findings that address postoperative management of patients with completely resected NSCLC. With regard to regular chest CT scans, Expert Opinion 10 noted that IFCT-0302 is the only prospective randomized multicenter study that focuses on this issue; the results of this study were presented at the European Society for Medical Oncology 2017 (6). The findings indicated that follow up with scheduled CT scans did not contribute to OS among patients with completely resected stage I-III NSCLC. Although this result was supported using data collected from 1,775 study patients, it is also true that most of the study patients were diagnosed with stage I disease $(68.1 \%)$; as such, it is not clear whether the results of this study are truly valid for patients with stage II-III NSCLC who have a more profound risk for recurrence.

At this time, we have no data on the benefits provided by annual brain MRI or bone scans. Local treatments, including Gamma Knife surgery and surgical resection remain promising and as such, early detection of brain metastasis might facilitate these local treatments and contribute to quality of life. Given these issues, brain MRIs scheduled according to risk for recurrence may be well worth considering in our clinical practice. However, as noted by Expert Opinion 7, categorizing this recommendation as "strong" seems to be a bit too high given the absence of any clear evidence-based data focused on this issue, particularly for the subset of EGFR-mutant NSCLCs.

Consensus 8 states: "in EGFR-mutant NSCLC patients with postoperative recurrence and metastasis, EGFR-TKI (preferably osimertinib) can be a treatment choice for salvage therapy (level of evidence: 1 ; strength of recommendation: strong)." This recommendation is fully appropriate and based on evidence from the FLAURA study; most of Expert Opinions also agreed $(7,8)$. As noted by Expert Opinion 3, most of the patients in the FLAURA study were diagnosed with stage IV disease, but not postoperative recurrence. The management of postoperative recurrence will inevitably be linked to that of stage IV disease, as it is unlikely that a prospective randomized study that includes only those patients with postoperative recurrence will be performed. As such, drug therapy for postoperative recurrence of EGFR-mutant NSCLC will ultimately be aligned for stage IV disease both now and in the future.

The eight recommendations in this report are thoughtful and give us some hints in practice of managing surgically resected EGFR-mutant NSCLC. However, there are some differences in frequency of EGFR mutation, rule about proper usage of drugs and the system of medical treatment insurance, in different countries. We should adapt successfully these recommendations to our practical condition from the varying viewpoints.

\section{Acknowledgments}

Funding: None. 


\section{Footnote}

Provenance and Peer Review: This article was commissioned by the editorial office, Annals of Translational Medicine. The article did not undergo external peer review.

Conflicts of Interest: All authors have completed the ICMJE uniform disclosure form (available at http://dx.doi. org/10.21037/atm.2020.04.34). The authors have no conflicts of interest to declare.

Ethical Statement: The authors are accountable for all aspects of the work in ensuring that questions related to the accuracy or integrity of any part of the work are appropriately investigated and resolved.

Open Access Statement: This is an Open Access article distributed in accordance with the Creative Commons Attribution-NonCommercial-NoDerivs 4.0 International License (CC BY-NC-ND 4.0), which permits the noncommercial replication and distribution of the article with the strict proviso that no changes or edits are made and the original work is properly cited (including links to both the formal publication through the relevant DOI and the license). See: https://creativecommons.org/licenses/by-nc-nd/4.0/.

\section{References}

1. Dearden S, Stevens J, Wu YL, et al. Mutation incidence and coincidence in non small-cell lung cancer: metaanalyses by ethnicity and histology (mutMap). Ann Oncol

Cite this article as: Nakao M, Ichinose J, Matsuura Y, Okumura S, Mun M. Different perspectives and viewpoints on the postoperative management of EGFR-mutant lung cancer. Ann Transl Med 2020;8(18):1201. doi: 10.21037/ atm.2020.04.34
2013;24:2371-6.

2. Liang W, Cai K, Chen C, et al. Society for Translational Medicine consensus on postoperative management of EGFR-mutant lung cancer (2019 edition). Transl Lung Cancer Res 2019;8:1163-73.

3. Kelly K, Altorki NK, Eberhardt WE, et al. Adjuvant Erlotinib Versus Placebo in Patients With Stage IBIIIA Non-Small-Cell Lung Cancer (RADIANT): A Randomized, Double-Blind, Phase III Trial. J Clin Oncol 2015;33:4007-14.

4. Zhong WZ, Wang Q, Mao WM, et al. Gefitinib versus vinorelbine plus cisplatin as adjuvant treatment for stage II-IIIA (N1-N2) EGFR-mutant NSCLC (ADJUVANT/ CTONG1104): a randomised, open-label, phase 3 study. Lancet Oncol 2018;19:139-48.

5. Yue D, Xu S, Wang Q, et al. Erlotinib versus vinorelbine plus cisplatin as adjuvant therapy in Chinese patients with stage IIIA EGFR mutation-positive non-small-cell lung cancer (EVAN): a randomised, open-label, phase 2 trial. Lancet Respir Med 2018;6:863-73.

6. Westeel V, Barlesi F, Foucher P, et al. Results of the phase III IFCT-0302 trial assessing minimal versus CT-scanbased follow-up for completely resected non-small cell lung cancer (NSCLC). Ann Oncol 2017;28:452.

7. Soria JC, Ohe Y, Vansteenkiste J, et al. Osimertinib in Untreated EGFR-Mutated Advanced Non-Small-Cell Lung Cancer. N Engl J Med 2018;378:113-25.

8. Ramalingam SS, Vansteenkiste J, Planchard D, et al. Overall Survival with Osimertinib in Untreated, EGFR-Mutated Advanced NSCLC. N Engl J Med 2020;382:41-50. 